01

\title{
Расчетно-экспериментальное исследование ударно-волнового нагружения оптически прозрачных тел
}

\author{
() С.И. Герасимов, ${ }^{1-4}$ В.А. Кузьмин, ${ }^{1,2,4}$ В.А. Кикеев, ${ }^{3,4}$ Н.А. Трепалов ${ }^{1}$ \\ ${ }^{1}$ РФЯЦ-ВНИИ экспериментальной фризики, \\ 607190 Саров, Нижегородская обл., Россия \\ ${ }^{2}$ Саровский физико-технический институт — филиал НИЯУ МИФИ, \\ 607190 Саров, Нижегородская обл., Россия \\ ${ }^{3}$ Нижегородский государственный технический университет им. Р.Е. Алексеева, \\ 603950 Нижний Новгород, Россия \\ ${ }^{4}$ Институт проблем машиностроения РАН - фрилиал Института прикладной фризики РАН, \\ 603024 Нижний Новгород, Россия \\ e-mail: s.i.gerasimov@mail.ru, v_a_kuzmin@mail.ru, vkikeev@mail.ru, natrepalov@gmail.com
}

Поступило в Редакцию 22 августа 2018 г.

В окончательной редакции 22 августа 2018 г.

Принято к публикации 11 марта 2019 г.

\begin{abstract}
Представлены результаты расчетного и экспериментального исследований процессов ударно-волнового нагружения прозрачных тел двумя методами: теоретическим - на основе численного моделирования на регулярной трехмерной сетке с использованием явного решателя в связной лагранжево-эйлеровой постановке, экспериментальным - с использованием метода теневого фотографирования и теневого фонового метода.
\end{abstract}

Ключевые слова: визуализация, ударно-волновое нагружение, метод численного решения, теневая фоторегистрация, теневой фоновый метод.

DOI: 10.21883/JTF.2019.09.48056.323-18

\section{Введение}

Изучение реакции твердых тел на ударно-волновое нагружение является важной и сложной задачей. Определение характера и времени распространения волновых возмущений позволяет дать заключение о физикомеханических свойствах нагружаемого объекта, подобрать константы и провести верификацию математической модели. Для этих целей применяются различные экспериментальные методы: метод Кольского, методы с использованием манганиновых датчиков, а также методы визуализации быстропротекающих процессов.

Для визуализации ударно-волновых процессов в оптически прозрачных объектах применяются методы, основанные на зависимости показателя преломления прозрачных сред от локальной плотности среды - теневые методы. В непрозрачных телах волновой портрет на заданный момент времени содержит набор ударных волн над поверхностью противоположной нагружаемой - результат преломления волн сжатия, циркулирующих в нагруженном материале, на свободной границе с воздухом. Кроме того, выход сильной ударной волны на свободную границу может сопровождаться откольными явлениями, либо пылением (ejecta), что также визуализируется теневым методом регистрации.

Вместе с тем необходимо отметить неизменно возрастающую роль численного моделирования, средств компьютерной обработки и представления результатов в изучении быстропротекающих процессов. Сейчас чаще всего именно комплексное использование экспериментальных методов и средств получения необходимой информации о том или ином физическом процессе и численного моделирования с широкими возможностями представления полученных результатов расширяет инструментарий исследователя [1].

В настоящей работе показаны результаты применения схемы теневой фоторегистрации - прямотеневое фотографирование на низкочувствительную пленку с применением взрывного источника света с отсечкой излучения за счет перемешивания [2]. Приведены экспериментальные картины развития волнового возмущения в образце из оргстекла и процессов, сопровождающих выход ударной волны на границу стальной пластины, а также результаты численного моделирования. Численное моделирование осуществлялось на регулярной трехмерной сетке с использованием явного решателя в связной лагранжево-эйлеровой постановке. На основе сравнения экспериментальных теневых картин распространения возмущения с расчетными данными определены упругие характеристики оргстекла.

Для представления результатов численного расчета и визуализации используются средства и возможности графического интерфейса [3]. 


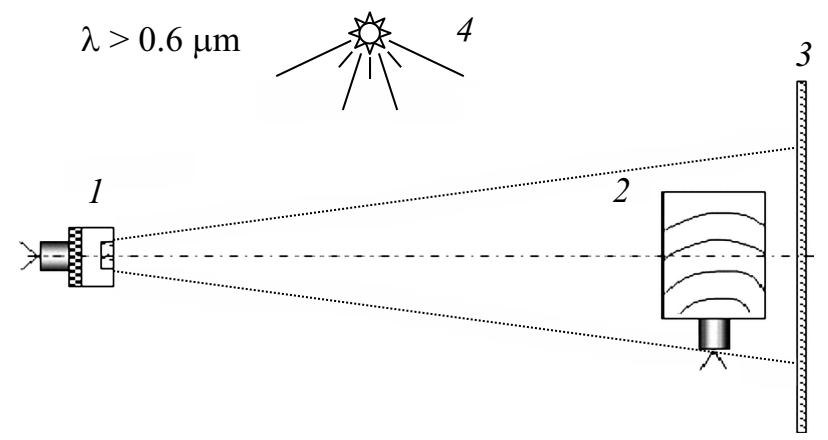

Рис. 1. Принципиальная схема размещения экспериментального оборудования: 1 - миниатюрный взрывной источник света, 2 - объект исследования, 3 - низкочувствительная фотопленка, 4 - искусственное освещение.

\section{1. Визуализация процессов ударно-волнового нагружения плиты из оргстекла}

\section{1. Постановка эксперимента}

Объектом исследования является плита из оргстекла с размерами $55 \times 55 \times 10 \mathrm{~mm}$. Нагружение осуществлялось взрывом электродетонатора, который располагался снизу, контактируя с центром нижней грани. Принципиальная схема размещения [2] экспериментального оборудования и образца приведена на рис. 1.

На основе полученных в различные моменты времени фотографий можно сделать вывод о скорости, характере распространения возмущения по образцу, и подобрать физико-механические свойства математической модели.

\section{2. Численное моделирование}

Численный расчет ударно-волнового нагружения проводился в конечноэлементном программном комплексе с использованием явного решателя [4]. Решение осуществлялось произвольным лагранжево-эйлеровым методом $[5,6]$ на регулярной трехмерной сетке. Использовались трехмерные восьмиузловые элементы Solid 164 с заданием формулировки Лагранжа для описания плиты из оргстекла, и формулировки Эйлера для описания тех частей, которые ведут себя как жидкость - воздух и взрывчатое вещество (BВ). Вид расчетной модели показан на рис. 2.

Размер ячейки расчетной области, занимаемой оргстеклом, составляет $0.76 \mathrm{~mm}$. Количество ячеек, описывающих объем оргстекла, составляет 72576. Общее количество ячеек, принятое для описания данной задачи, составляет 268654.
Система уравнений, описывающих течение среды [7], имеет вид

$$
\left\{\begin{array}{l}
\frac{\partial \rho}{\partial t}+\nabla \rho(\mathbf{v}-\mathbf{u})+\rho \operatorname{div} \mathbf{v}=0 \\
\rho \frac{\partial \mathbf{v}}{\partial t}+\rho \nabla \mathbf{v}(\mathbf{v}-\mathbf{u})=\operatorname{div} \sigma \\
\rho \frac{\partial E}{\partial t}+\rho \nabla E(\mathbf{v}-\mathbf{u})=\sigma \dot{\varepsilon}
\end{array}\right.
$$

где $\rho$ - плотность среды, $\mathbf{v}$ - вектор скорости среды, и - вектор скорости узлов сетки, $\sigma$ - тензор напряжений, $\dot{\varepsilon}-$ тензор скоростей деформаций, $E-$ внутренняя энергия, $t-$ текущее время.

Напряженно-деформированное состояние в точке расчетной области определялось общей системой уравнений:

$$
\begin{gathered}
\sigma_{i j}=-p \delta_{i j}+s_{i j}, \\
p=p(\rho, E),
\end{gathered}
$$

где $\sigma_{i j}-$ тензор напряжений, $s_{i j}-$ девиатор напряжений, $\delta_{i j}$ - символ Кронекера, $p$ - гидростатическое давление, заданное уравнением состояния (УРС).

Каждой части расчетной модели ставится в соответствие своя математическая модель материала, позволяющая адекватно описать заданное воздействие.

Плита из оргстекла описывалась упругой моделью материала в соответствии с системой уравнений

$$
\begin{gathered}
s_{i j}=2 G e_{i j}, \\
p=-K \theta,
\end{gathered}
$$

где $G$ - модуль сдвига материала, $s_{i j}-$ девиатор напряжений, $p$ - гидростатическое давление, $e_{i j}-$ девиатор деформаций, $\theta$ - объемная деформация.

Воздух в расчете подразумевался идеальной средой без учета вязкостных свойств. Напряженнодеформированное состояние определяется только гидро-

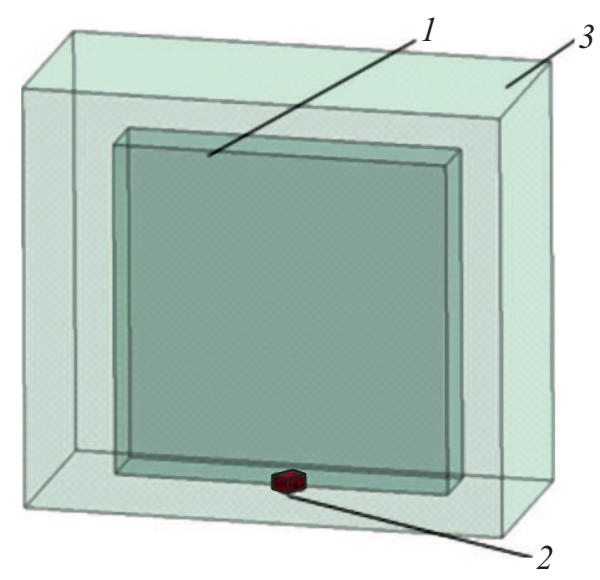

Рис. 2. Расчетная модель: 1 - пластина из оргстекла, 2 - электродетонатор (слой ВВ), 3 - объем воздуха. 
Таблица 1. Физико-механические свойства оргстекла

\begin{tabular}{c|c|c}
\hline $\begin{array}{c}\text { Плотность, } \\
\mathrm{kg} / \mathrm{m}^{3}\end{array}$ & $\begin{array}{c}\text { Модуль упругости } E, \\
\mathrm{MPa}\end{array}$ & $\begin{array}{c}\text { Коэффициент } \\
\text { Пуассона } v\end{array}$ \\
\hline 1180 & 7000 & 0.3
\end{tabular}

Таблица 2. Параметры модели материала ВВ

\begin{tabular}{c|c|c}
\hline $\begin{array}{c}\text { Плотность ВВ } \\
\rho, \mathrm{kg} / \mathrm{m}^{3}\end{array}$ & $\begin{array}{c}\text { Скорость детонации } \\
D, \mathrm{~km} / \mathrm{s}\end{array}$ & $\begin{array}{c}\text { Давление Чепмена-Жуге } \\
P_{C J}, \mathrm{GPa}\end{array}$ \\
\hline 1717 & 7.98 & 29.5
\end{tabular}

Таблица 3. Параметры уравнения состояния продуктов детонации $\mathrm{BB}$

\begin{tabular}{c|c|c|c|c}
\hline$A, \mathrm{GPa}$ & $B, \mathrm{GPa}$ & $R_{1}$ & $R_{2}$ & $\omega$ \\
\hline 524.2 & 7.678 & 4.2 & 1.1 & 0.34
\end{tabular}

статическим давлением

$$
p=(k-1) \frac{\rho}{\rho_{0}} E,
$$

где $k=1.4$ - показатель адиабаты, $E-$ внутренняя энергия на единицу объема, $\rho_{0}$ - начальная плотность.

Для задания материала ВВ использовалась специальная модель ВВ, позволяющая моделировать детонацию и течение продуктов взрыва $[4,5]$. Давление в элементе ВВ в каждый момент времени определяется по формуле

$$
p=F p(\rho, E),
$$

где $F=\max \left(F_{1}, F_{2}\right)$ - доля выгорания $\mathrm{BB}$,

$$
F_{1}=\frac{\rho D^{2}}{P_{C J}}\left(1-\frac{V}{V_{0}}\right), F_{2}=\frac{2 D\left(t-t_{b}\right)}{3 \Delta x},
$$

$\rho$ - плотность ВВ, $D-$ скорость детонации, $P_{C J}-$ давление Чепмена-Жуге, $t_{b}$ - время выгорания элемента, $\Delta x$ - характерный размер элемента.

УРС для продуктов детонации ВВ принято в форме Джонса-Уилкинса-Ли $[4,8]$ :

$$
\begin{aligned}
p(\rho, E)= & A\left(1-\frac{\varpi}{R_{1} V}\right) e^{-R_{1} V} \\
& +B\left(1-\frac{\varpi}{R_{1} V}\right) e^{-R_{2} V}+\frac{\varpi E}{V},
\end{aligned}
$$

где $V=\frac{\rho_{0}}{\rho}$ - относительный объем продуктов детонации ВВ в процессе взрыва, $A, B, R_{1}, R_{2}, \omega$ - эмпирические константы, $E-$ внутренняя энергия в единице объема, $\varpi=-\left(\frac{\partial \ln p}{\partial \ln V}\right)_{S}-1-$ при расширении продуктов взрыва до величины $V>10, S$ - энтропия.
Уравнению (6) соответствует изоэнтропа продуктов взрыва:

$$
p_{s}=A e^{-R_{1} V}+B e^{-R_{2} V}+C V^{-(1+\varpi)} .
$$

Упругие свойства оргстекла и плотность приведены в табл. 1.

Параметры УРС ВВ взяты в соответствии с [9] и приведены в табл. 3 .

Плотность оргстекла составляла $\rho_{0}=1180 \mathrm{~kg} / \mathrm{m}^{3}$. Модуль упругости и коэффициент Пуассона подобраны таким образом, чтобы волновая картина численного расчета совпадала с экспериментом.

Плотность воздуха составляла $\rho_{0}=1.225 \mathrm{~kg} / \mathrm{m}^{3}$.

Параметры модели материала ВB, принятые в соответствии с [9], приведены в табл. 2.

\section{3. Результаты численного моделирования и эксперимента}

На приведенных ниже рисунках представлены картины распространения ударно- волнового возмущения по плите из оргстекла. На теневой фотографии это выражено в регистрации оптических неоднородностей при выходе возмущений на свободную поверхность и зоны потери прозрачности в объеме оргстекла. Рядом с теневыми фотографиями приведены результаты численного расчета в виде полей скорости и давления.

Чтобы выполнить сравнение результатов численного расчета с результатами эксперимента, выбиралось распределение давлений и скоростей эквивалентное распределению зон потери прозрачности оргстекла. Критерием правильности выбора параметров расчетной модели, описывающей оргстекло, было совпадение моментов времени экспериментальных картин и расчетных.

На рис. 3 помимо теневых фотографий представлены картины распределения полей давления и результирующей скорости. Процесс распространения возмущения и в эксперименте и в численной реализации начинается от нижней грани образца после приложения импульсной нагрузки - взрыва капсюля-детонатора (заряда взрывчатого вещества). В процессе распространения волнового возмущения происходит многократное отражение волны от свободной нижней грани с формированием сложной картины. С течением времени отражение происходит от боковых и верхней граней. Это показано на рис. 3, где приведена теневая картина возмущений на момент времени $12 \mu \mathrm{s}$ и соответствующие теневой картине возмущения, полученные в численном расчете на момент времени $12.2 \mu \mathrm{s}$. Неабсолютное совпадение времен связано с погрешностью численного расчета. Качественно лучшее совпадение с экспериментом наблюдается у картин с распределением скоростей ввиду того, что затемнения на теневой картине соответствуют горбам и впадинам на поверхности плиты из оргстекла, т.е. колебательному характеру распространения возмущения. А колебательный процесс в данном численном 


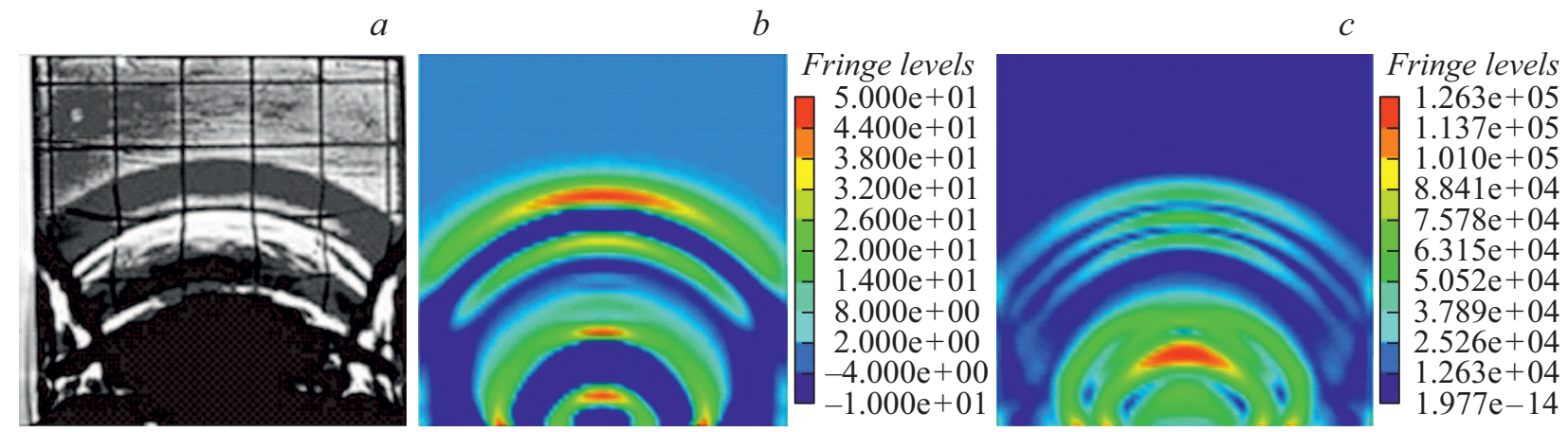

Рис. 3. Визуализация процесса: $a-$ теневая картина процесса; $b-$ распределение давления, $[\mathrm{MPa}] ; c-$ распределение результирующей скорости, $[\mathrm{mm} / \mathrm{s}]$.

эксперименте лучше всего визуализировать, отображая скорость точек модели.

Как видно из картин, представленных на рис. 3, максимальная погрешность временной реализации волновой картины в расчете по сравнению с экспериментом составляет не больше $3.2 \%$.

Об осцилляции свободной поверхности можно судить по графику изменения скорости точки, расположенной на свободной поверхности плиты. Точки, в которых определялась скорость, показаны на рис. 4. Графики изменения скоростей соответствующих точек показаны на рис. 5 и 6.

По графикам изменения скоростей точек можно сделать вывод, что на момент времени $t=30 \mu \mathrm{s}$ боковая поверхность (где расположены точки $A$ и $B$ ) совершила 6 осцилляций, верхняя (где расположена точка $C$ ) 5 осцилляций. Это соответствует теневой картине.

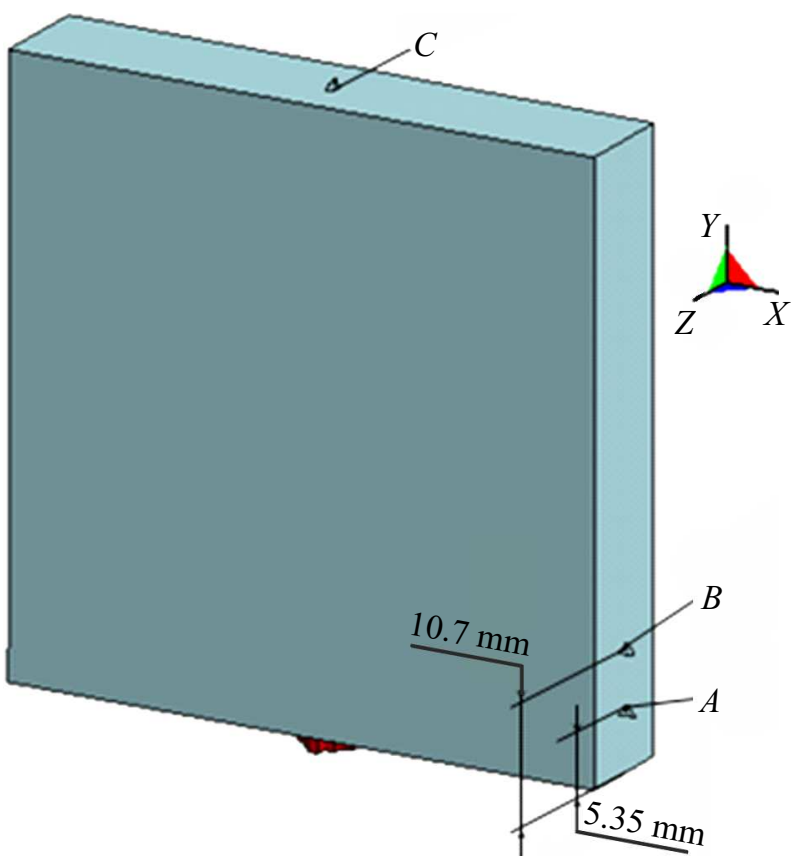

Рис. 4. Точки определения скорости.

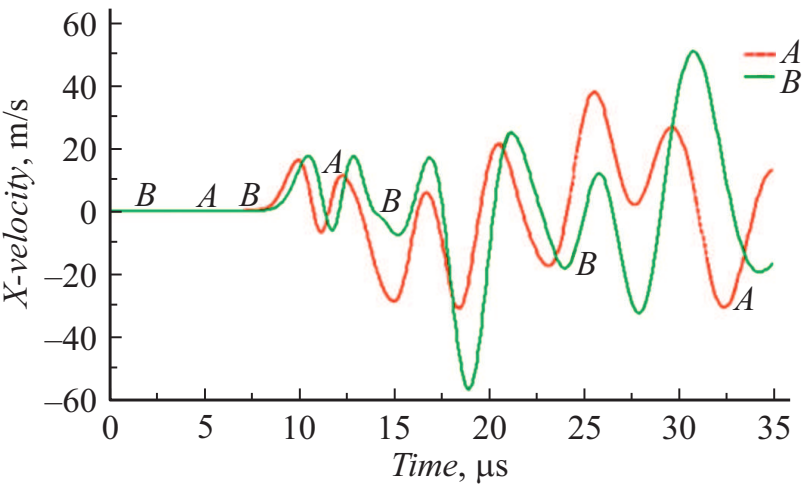

Рис. 5. Скорости точек $A$ и $B$ в направлении оси $X$ (см. рис. 4).

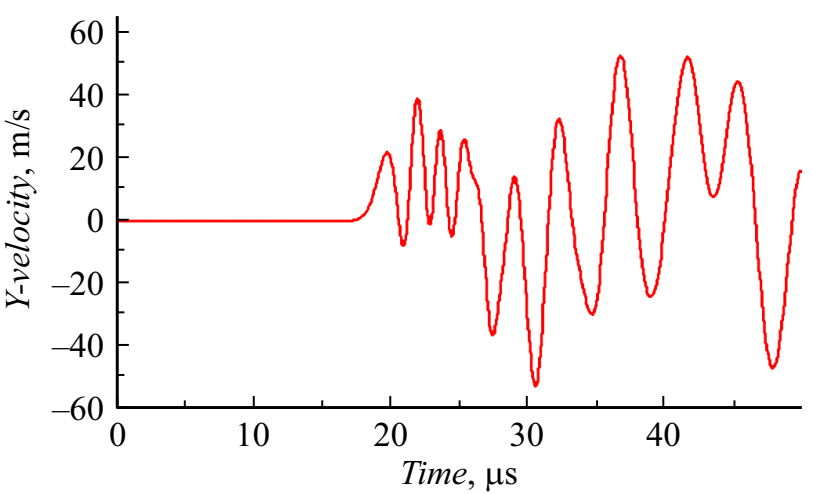

Pис. 6. Скорости точки $C$ в направлении оси $Y$ (см. рис. 4).

\section{2. Визуализация процесса ударно-волнового нагружения массивного тела из оргстекла}

В разд. 2 приводятся результаты регистрации распространения ударно-волнового возмущения в объеме оргстекла теневым фоновым методом, а также результаты численного моделирования. Численное моделирование, также как и в предыдущем разделе, осуществлялось на регулярной трехмерной сетке с использованием явного решателя в связной лагранжево-эйлеровой постановке. 


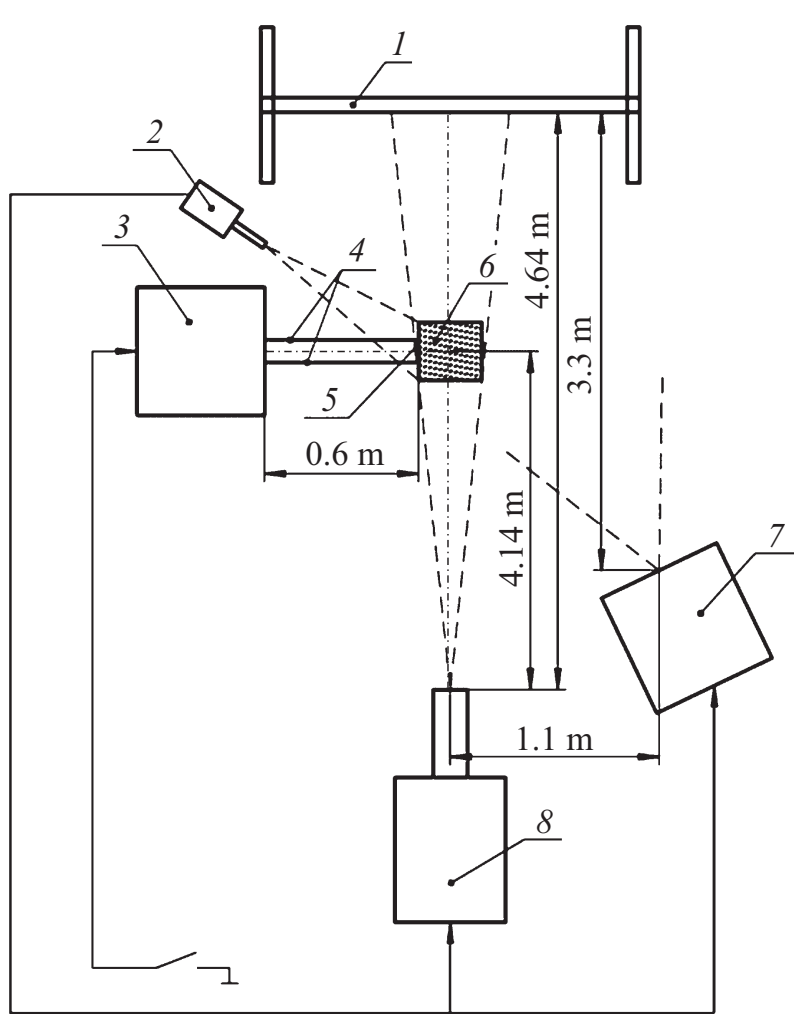

Рис. 7. Принципиальная схема размещения экспериментального оборудования: 1 - фоновый экран, $2-$ фотоприемное устройство, 3 - накопитель энергии, 4 - электроды, 5 - проволочка, 6 - оргстекло, 7 - импульсный источник света, 8 - электронно-оптическая камера „Наногейт 22/2“.

\section{1. Постановка эксперимента}

Объектом исследования является плита из оргстекла с размерами $155 \times 150 \times 105 \mathrm{~mm}$. Нагружение осуществлялось взрывом медной проволочки диаметром $0.09 \mathrm{~mm}$ и длиной $28 \mathrm{~mm}$. В качестве источника высокого напряжения использовался накопитель с энергией разрядного контура $216 \mathrm{~J}$ и уровнем напряжения $12 \mathrm{kV}$.

Принципиальная схема размещения экспериментального оборудования и образца приведена на рис. 7.

Характерный размер ячейки расчетной области, занимаемой оргстеклом, составляет $1 \mathrm{~mm}$. Количество ячеек, описывающих объем оргстекла, составляет 2441250. Общее количество ячеек, принятое для описания данной задачи, составляет 3161200.

На основе полученных в различные моменты времени фотографий можно сделать вывод о скорости, характере распространения возмущения по образцу, и подобрать физико-механические свойства математической модели.

\section{2. Численное моделирование}

Численный расчет ударно-волнового нагружения проводился в конечно-элементном программном комплексе с использованием явного решателя [4]. Решение
Таблица 4. Параметры модели материала ВВ

\begin{tabular}{c|c|c}
\hline $\begin{array}{c}\text { Плотность ВВ } \\
\rho, \mathrm{kg} / \mathrm{m}^{3}\end{array}$ & $\begin{array}{c}\text { Скорость детонации } \\
D, \mathrm{~km} / \mathrm{s}\end{array}$ & $\begin{array}{c}\text { Давление Чепмена-Жуге } \\
P_{C J}, \mathrm{GPa}\end{array}$ \\
\hline 1000 & 5.1 & 7500
\end{tabular}

Таблица 5. Параметры уравнения состояния продуктов детонации ВB

\begin{tabular}{c|c|c|c|c}
\hline$A, \mathrm{GPa}$ & $B, \mathrm{GPa}$ & $R_{1}$ & $R_{2}$ & $\omega$ \\
\hline 162.7 & 10.82 & 5.4 & 1.4 & 0.25
\end{tabular}

осуществлялось так же, как и в предыдущей задаче лагранжево-эйлеровым $[5,6]$ методом на регулярной трехмерной сетке. Взрыв проволочки моделировался взрывом тонкой полоски ВВ с размерами $1.5 \times 1.0 \times 30 \mathrm{~mm}$ с энерговыделением $200 \mathrm{~J}$.

Математический аппарат, лежащий в основе численных алгоритмов, и модели материалов, использованных при решении этой задачи, описаны в разд. 1.2.

Поскольку взрыв проволочки заменен взрывом ВВ с энерговыделением $200 \mathrm{~J}$, были подобраны соответствующие параметры ВВ, которые приведены в табл. 4 и 5.

Параметры модели материала ВВ с необходимым энерговыделением, принятые в соответствии с [9], приведены в табл. 4.

Параметры УРС ВВ взяты в соответствии с [9] и приведены в табл. 5 .

\section{3. Результаты численного моделирования и эксперимента}

На приведенном ниже рис. 8 представлены экспериментальная и расчетная картины распространения ударно-волнового возмущения в объеме оргстекла. Экспериментальная картина получена с помощью теневого фонового метода, в результате обработки и сопоставления невозмущенного и возмущенного изображений фонового экрана. Рядом с экспериментальной картиной

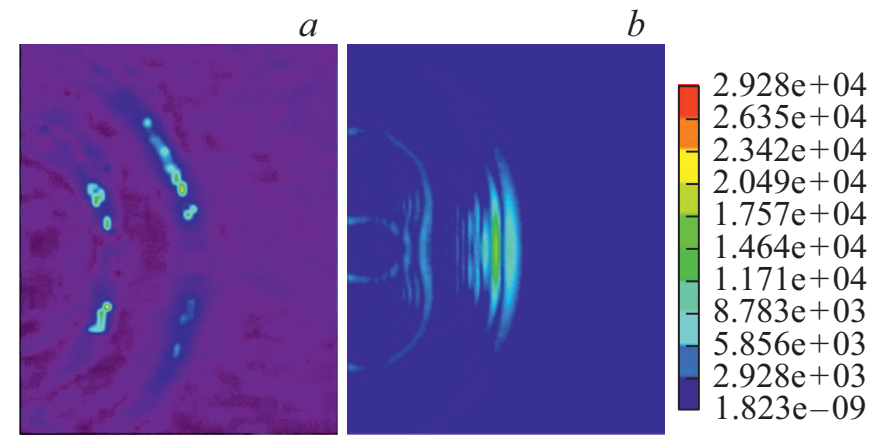

Рис. 8. $a-$ экспериментальная картина процесса, $b-$ pacпределение результирующей скорости, $[\mathrm{mm} / \mathrm{s}]$. 
показана картина, полученная в результате численного расчета в виде поля скорости.

Так же как в разд. 1.3 для сравнения результатов численного расчета с результатами эксперимента выбиралось распределение скорости, эквивалентное картине, сделанной теневым фоновым методом. Критерием правильности выбора параметров расчетной модели, описывающей оргстекло, было совпадение моментов времени экспериментальных картин и расчетных.

\section{Заключение}

1. В работе приведены теневые фотографии и фотографии, сделанные с помощью теневого фонового метода, ударно-волнового нагружения оргстекла. Представлены результаты численного моделирования в связанной лагранжево-эйлеровой постановке, в котором оргстекло описывалось упругой моделью материала. Упругие константы модели подобраны итерационно из соответствия волновых картин в различные моменты времени экспериментальным данным, а именно модуль упругости $E=7000 \mathrm{MPa}$, коэффициент Пуассона $v=0.3$. По данным экспериментов проведена верификация математической модели, которая показала хорошее соответствие расчетной модели полученным экспериментальным данным.

2. Показано, что имея результаты оптической регистрации процесса распространения ударно-волнового возмущения, не прибегая к дополнительным инструментариям, например емкостным датчикам скорости, и используя возможности современных программных комплексов численного моделирования, можно идентифицировать некоторые константы материала экспериментального образца, а также провести верификацию математических моделей, описывающих деформирование материала образцов.

Численное моделирование выполнено на базе НГТУ им. Р.Е. Алексеева

\section{Финансирование работы}

Исследование выполнено при финансовой поддержке РФФИ (грант 18-38-00297).

\section{Конфликт интересов}

Авторы заявляют, что у них нет конфликта интересов.

\section{Список литературы}

[1] Пилюгин В., Маликова Е. Пасько А., Аджсиев В. // Научная визуализация. 2012. Т. 4. № 4. С. 56-70.

[2] Герасимов С.И. Теневое фотографирование в расходящемся пучке света. Монография. Саров: ФГУП „РФЯЦВНИИЭФ“, 2010.
[3] Кравчук А.С., Чашинский А.С., Кравчук А.И. Основные элементы графического интерфейса LS-PREPOST. Минск: БГУ, 2013.

[4] Hallquist J.O. LS-DYNA: Theoretical manual. Livermore Software Technology Corporation. Livermore, 1998.

[5] Souli M. LS-Dyna Advanced Course in ALE and Fluid/Structural Coupling. Course Note for Arbitrary Lagrangian-Eulerian Formulation Technique. Livermore, LSTC, CA, 2000.

[6] Белоиерковский О.М., Давыдов Ю.М. Метод крупных частиц в газовой динамике. М.: Наука, 1982.

[7] Муйземнек А.Ю., Богач А.А. Математическое моделирование процессов удара и взрыва в программе LS-DYNA. Пенза: Информационно-издательский центр ПГУ, 2005.

[8] Lee E., Finger M., Collins $W$. JWL equation of state coefficients for high explosives. Rept-UCID-16189, Lawrence Livermore National Laboratory, 1973.

[9] Орленко Л.П. Физика взрыва. Изд. 3-е, испр. В 2 т. Т. 1. М.: Физматлит, 2004. 